\title{
Infective Endocarditis Caused by C. sordellii: The First Case Report from India
}

\author{
Rama Chaudhry ${ }^{1}$ Tej Bahadur ${ }^{1}$ Tanu Sagar ${ }^{1}$ Sonu Kumari Agrawal ${ }^{1} \quad$ Nazneen Arif ${ }^{1}$ \\ Shiv K. Choudhary² Nishant Verma ${ }^{1}$
}

${ }^{1}$ Department of Microbiology, All India Institute of Medical Sciences, New Delhi, India

${ }^{2}$ Department of Cardiothoracic and Vascular Surgery, All India Institute of Medical Sciences, New Delhi, India

Address for correspondence Dr. Rama Chaudhry, MD, Department of Microbiology, All India Institute of Medical Sciences, Ansari Nagar, New Delhi 110029, India (e-mail: drramach@gmail.com).

J Lab Physicians 2021;13:74-76.
Abstract
Keywords
- infective endocarditis
- Clostridium sordellii
- diagnosis

Clostridium sordellii is a gram-positive anaerobic bacteria most commonly isolated from skin and soft tissue infection, penetrating injurious and intravenous drug abusers. The exotoxins produced by the bacteria are associated with toxic shock syndrome. We report here a first case of infective endocarditis due to $C$. sordellii from a female patient with ventricular septal defect from India.

\section{Introduction}

Anaerobes are major components of the normal microbial flora present on human skin and mucosa. Infections due to anaerobic bacteria are common, but they are difficult to isolate from infected sites and are often overlooked. ${ }^{1}$ Anaerobic endocarditis is a rare entity. Aerobes such as Staphylococcus aureus, Streptococcus viridians, groups A, C, and G streptococci and Enterococcus species are the most common microorganisms that cause infective endocarditis (IE). ${ }^{1}$

The most frequently reported causes of anaerobic endocarditis are Bacteroides (particularly the Bacteroides fragilis group), anaerobic Streptococcus, Clostridium spp., Peptostreptococcus, Fusobacterium, Propionibacterium, and Lactobacillus spp. ${ }^{2}$ Clostridium sordellii endocarditis has not been reported from India. Here, we report the first case of C. sordellii endocarditis in a female patient from India.

\section{Case Details}

A 28-year-old female, suffering from acyanotic congenital heart disease with ventricular septal defect (VSD) was admitted to the cardiothoracic vascular surgery ward of All India Institute of Medical Sciences (AIIMS), New Delhi, India with complaints of worsening shortness of breath and palpitations. Patient reported having an episode of IE 3 months back for which she had been admitted to AIIMS and was discharged after treatment. However, with the latest presentation there was no history of fever, although there was a history of tooth extraction a week before.

On clinical examination, there was a loud diastolic murmur in the aortic area. The chest roentgenogram revealed cardiomegaly. An echocardiography showed a ruptured sinus of Valsalva with normal right ventricular function, severe aortic regurgitation, and no evidence of cardiac vegetations. Hemogram revealed a hemoglobin of $9.6 \mathrm{~g} / \mathrm{dL}$ with a platelet count of $120,000 / \mathrm{mm}^{3}$. Urine and blood cultures of the patient were consistently sterile. Within 5 days of admission, an RSOV repair was performed. The VSD was closed with a Gor-Tex patch and an aortic valve replacement was done. Aortic valve tissues were sent for aerobic, anaerobic, and fungal culture. The postoperative period was uneventful.

On microscopy, there was no evidence of bacteria on Gram Stain or fungal elements on $\mathrm{KOH}$ mount. Cultures on routine and enriched bacteriological media were consistently published online May 19, 2021
DOI https://doi.org/

$10.1055 / \mathrm{s}-0041-1723750$

ISSN 0974-2727. (c) 2021. The Indian Association of Laboratory Physicians.

This is an open access article published by Thieme under the terms of the Creative Commons Attribution-NonDerivative-NonCommercial-License, permitting copying and reproduction so long as the original work is given appropriate credit. Contents may not be used for commercial purposes, or adapted, remixed, transformed or built upon. (https://creativecommons.org/licenses/by-nc-nd/4.0/).

Thieme Medical and Scientific Publishers Pvt. Ltd. A-12, 2nd Floor, Sector 2, Noida-201301 UP, India 
negative, even on prolonged aerobic incubation. Aerobic blood cultures sent previously also had been consistently negative.

The specimen was received in Robertson's cooked meat (RCM) medium in anaerobic bacteriology laboratory. After 48 hours of RCM incubation under anaerobic conditions, subcultures were performed on Brain Heart Infusion Blood Agar (BHIBA), Blood Agar (BA), and MacConkey Agar (MA). The BHIBA was incubated under anaerobic conditions and $B A$ in $\mathrm{CO}_{2}$ desiccator as per standard protocol. ${ }^{2} \mathrm{BHIBA}$ plates showed the growth of metronidazole sensitive colonies which upon Gram staining revealed gram-positive bacilli with subterminal spores. BA and MA showed no growth. Conventional biochemicals were set up after obtaining growth anaerobically in peptone yeast extract broth. Organism produced indole and urease, liquefied gelatin, fermented glucose, and produced lecithinase. It did not ferment lactose, hydrolyze esculin, reduce nitrates and was lipase negative. The findings were confirmed using the API $20 \mathrm{~A}$ system. It was labeled as $C$. sordellii based on the findings. After the identification, we further inquired about the history of diarrhea to rule out any translocation of Clostridioides difficile (morphologically similar organism) from gastrointestinal tract into the blood stream and no such history was obtained. The isolate $C$. sordellii was subjected to polymerase chain reaction (PCR) for $C$. difficile toxins $\mathrm{A}$ and $\mathrm{B}$ (tcdA and tcdB) and was found to be negative. ${ }^{3}$ The patient was put on metronidazole along with other antibiotics and was discharged after improvement. On follow-up, she was doing well and had no evidence of recurrence of infection.

\section{Discussion}

Although IE due to anaerobic bacteria is a rare condition, a surge in recent times has been observed. The increased use of interventional procedures for diagnosis or management predisposes individuals to develop bacteremia due to aerobic and anaerobic bacteria. ${ }^{3}$ In the year 1977, Finegold et al reported over 200 cases of anaerobic IE, out of which 17 cases were due to Clostridium species. He also showed that Clostridium perfringens was the commonest among all clostridia. ${ }^{4}$ Other species such as Clostridium bifermentans, Clostridium clostridiformis, Clostridium septicum, and Clostridium ramosum have also been implicated as a cause of IE ${ }^{5-7} \mathrm{C}$. sordellii IE has been scarcely reported.

It is thus evident from the above that Clostridia should be considered as a plausible cause of infectious endocarditis and not just dismissed as a laboratory contaminant upon being isolated from such cases. We also reviewed our laboratory data of last years. In the year 2019, we received 73 cardiac specimens of patients suffering from infectious endocarditis for anaerobic culture and found only one sample positive for Clostridium spp. Since $C$. sordellii's first isolation in the year 1922, it has been commonly linked with lethal posttraumatic skin and soft tissue infections, penetrating injury, medical abortions, pericarditis along with some reported cases of bacteremia (- Table 1). ${ }^{8-14}$ The pathogenicity of C. sordellii by productions of exotoxins are associated with toxic shock syndrome. ${ }^{15}$ Off these exotoxins, lethal toxin and hemorrhagic toxin are two major virulence factors, which showed antigenic as well as pathophysiological resemblance to $C$. difficile toxins $B$ and $A$, respectively. $C$. sordelli antitoxin is used to neutralize both $C$. difficile toxin $A$ and toxin $B$ by antigenic cross reactivity in cell culture assay. ${ }^{16}$

The abovementioned fact has been highlighted in a case report by Elsayed and Zhang ${ }^{17}$ in which a patient positive for C. difficile stool assay was actually suffering from $C$. sordellii infection. This signifies the fact that mere phenotypic characterization may lead to misdiagnosis. Both $C$. sordellii and $C$. difficile are asymptomatically carried in the gastrointestinal tracts of approximately $10 \%$ of adult humans and both share close similarity in host range and virulence factors; therefore, we performed molecular test to differentiate both. ${ }^{15,18}$

It is to be noted that emergence of the more virulent BI/ NAPI epidemic clone of $C$. difficile from various parts of United States of America and Canada further necessitates the need to carefully distinguish C. sordellii from C. difficile as an etiological agent as both the pathogens phenotypically may look similar. ${ }^{19}$ Therefore, the newer molecular techniques based on the detection of specific virulent genes will help to discriminate as was observed in this study where the strain was negative for C. difficile tcd A and B tox PCR. Thus, early and accurate identification of this organism is required for appropriate treatment as it is frequently misidentified as $C$. difficile species. This may lead to prescribing inappropriate antimicrobial therapy leading to mismanagement of the cases.

Table 1 Cases of Clostridium sordellii infection

\begin{tabular}{|l|l|l|l|l|l|}
\hline Age & Sex & Presenting complaints & Underlying condition & Outcome & $\begin{array}{l}\text { References and } \\
\text { year }\end{array}$ \\
\hline $67 \mathrm{y}$ & F & Septic shock & Pleomorphic gluteal sarcoma & Survived & $(2016)^{8}$ \\
\hline $8 \mathrm{mo}$ & - & Abscess in right thigh & Constrictive pericarditis & Survived & $(2011)^{9}$ \\
\hline $81 \mathrm{y}$ & F & Abdominal pain & Cholangitis & Survived & $(2011)^{10}$ \\
\hline $59 \mathrm{y}$ & F & Postoperative monitoring & Rectal malignancy & Fatal & $(2009)^{11}$ \\
\hline $27 \mathrm{y}$ & F & Pelvic pain and bleeding & Medical abortion & Fatal & $(2004)^{12}$ \\
\hline $81 \mathrm{y}$ & F & Perirectal necrosis & Traumatic self-evacuation & Fatal & $(2000)^{13}$ \\
\hline $12 \mathrm{y}$ & $\mathrm{M}$ & Ear infection & Epilepsy & Survived & $(2000)^{13}$ \\
\hline $73 \mathrm{y}$ & $\mathrm{M}$ & Perirectal abscess & Prostatic cancer & Fatal & $(1999)^{14}$ \\
\hline
\end{tabular}


Our report is aimed to draw attention to the accurate identification of $C$. sordellii in clinical samples, upon the isolation and identification as in this case in the microbiology laboratories.

In conclusion, $C$. sordellii is a rare infection, but one that needs a high index of suspicion to improve clinical outcome.

\section{Conflict of Interest}

None.

\section{References}

1 Brook I. Anaerobic infections in children. Microbes Infect 2002;4(12):1271-1280

2 Finegold SM, George WL, Mulligon ME, Anaerobic Infections. Chicago: Year book Medical Publishers; 1986

3 Cohen SH, Tang YJ, Silva J Jr. Analysis of the pathogenicity locus in Clostridium difficile strains. J Infect Dis 2000;181(2):659-663

4 Moreillon P, Que YA. Infective endocarditis. Lancet 2004;363(9403):139-149

5 Kolander SA, Cosgrove EM, Molavi A. Clostridial endocarditis. Report of a case caused by Clostridium bifermentans and review of the literature. Arch Intern Med 1989;149(2):455-456

6 Robles P, García-Gallego F, de Alba J, García J, Domínguez FJ, Oliver JM. Prosthetic endocarditis and splenic abscess caused by Clostridium clostridiforme [in Spanish]. Rev Esp Cardiol 1997;50(5):360-362

7 Muakkassa WF, Mohanty PK, Kipreous B, Lee HM, Goldman MH. Left ventricular mass with septic (Clostridium ramosum) arterial emboli in a renal allograft patient: report of a case and review of the literature. Transplant Proc 1983;15(2):1715-1719

8 Bonnecaze AK, Stephens SE, Miller PJ. Non-lethal Clostridium sordellii bacteraemia in an immunocompromised patient with pleomorphic sarcoma. BMJ Case Rep 2016;2016:2016

9 Chaudhry R, Verma N, Bahadur T, Chaudhary P, Sharma P, Sharma N. Clostridium sordellii as a cause of constrictive pericarditis with pyopericardium and tamponade. J Clin Microbiol 2011;49(10):3700-3702

10 Walk ST, Jain R, Trivedi I, et al. Non-toxigenic Clostridium sordellii: clinical and microbiological features of a case of cholangitis-associated bacteremia. Anaerobe 2011;17(5):252-256

11 Matten J, Buechner V, Schwarz R. A rare case of Clostridium sordellii bacteremia in an immunocompromised patient. Infection 2009;37(4):368-369

12 Wiebe E, Guilbert E, Jacot F, Shannon C, Winikoff B. A fatal case of Clostridium sordellii septic shock syndrome associated with medical abortion. Obstet Gynecol 2004;104(5 Pt 2):1142-1144

13 Abdulla A, Yee L. The clinical spectrum of Clostridium sordellii bacteraemia: two case reports and a review of the literature. J Clin Pathol 2000;53(9):709-712

14 Borer A, Gilad J, Sikuler E, Riesenberg K, Schlaeffer F, Buskila D. Fatal Clostridium sordellii ischio-rectal abscess with septicaemia complicating ultrasound-guided transrectal prostate biopsy. J Infect 1999;38(2):128-129

15 Aldape MJ, Bryant AE, Stevens DL. Clostridium sordellii infection: epidemiology, clinical findings, and current perspectives on diagnosis and treatment. Clin Infect Dis 2006;43(11):1436-1446

16 Popoff MR, Guillou JP, Carlier JP. Taxonomic position of lecithinase-negative strains of Clostridium sordellii. J Gen Microbiol 1985;131(7):1697-1703

17 Elsayed S, Zhang K. Positive Clostridium difficile stool assay in a patient with fatal C. sordellii infection. $\mathrm{N}$ Engl J Med 2006;355(12):1284-1285

18 Riggs MM, Sethi AK, Zabarsky TF, Eckstein EC, Jump RL, Donskey CJ. Asymptomatic carriers are a potential source for transmission of epidemic and nonepidemic Clostridium difficile strains among long-term care facility residents. Clin Infect Dis 2007;45(8):992-998

19 Curry SR, Marsh JW, Muto CA, O'Leary MM, Pasculle AW, Harrison LH. tcdC genotypes associated with severe TcdC truncation in an epidemic clone and other strains of Clostridium difficile. J Clin Microbiol 2007;45(1):215-221 